\title{
Terahertz-based system for dehydration analysis of hydrogel contact lenses
}

\author{
Francisco J. BURGOS-FERNÁNDEZ ${ }^{1}$, Ester GUAUS ${ }^{2 *}$, \\ CRistina MARTÍNEZ ${ }^{1}$, MeritXell VILASECA ${ }^{1}$ \\ ${ }^{1}$ Centre for Sensors, Instruments and Systems Development, Polytechnic University of Catalonia, \\ Rambla Sant Nebridi 10, 08222, Terrassa, Spain \\ ${ }^{2}$ Department of Chemical Engineering, Polytechnic University of Catalonia, \\ Colom 1, 08222, Terrassa, Spain
}

${ }^{*}$ Corresponding author: ester.guaus@upc.edu

\begin{abstract}
The use of terahertz-based techniques has grown very fast since they are capable of performing evaluations at molecular level, being very suitable for the analysis of biological samples and biomaterials such as those for contact lenses. These biomaterials are continuously evolving to enhance the lens wearer's comfort by improving their hydration state and surface wettability. Therefore, this study examines a novel terahertz system for the assessment of the temporary in vitro dehydration of hydrogel contact lenses, which provides a new index to assess their state of hydration. Several conventional and silicone hydrogel contact lenses and lens care solutions were analysed. Traditional methods such as the gravimetric determination of water content and the measurement of the static contact angle were also carried out for the validation of the developed system. The dehydration rate measurements of contact lenses obtained with the proposed system correlated with the values provided by traditional methods. As a whole, conventional hydrogel contact lenses exhibited the lowest values for dehydration rate. The tests conducted on various solutions showed a correlation between the wetting action of the solution and the dehydration rate of the contact lens material.
\end{abstract}

Keywords: terahertz radiation, terahertz systems, hydrogel contact lens dehydration, water content, lens care solutions.

\section{Introduction}

The use of new methods based on terahertz $(\mathrm{THz})$ radiation is growing fast in biomedical research, mainly because it is a non-invasive technique with non-ionizing properties [1]]. Terahertz radiation has a spectral range between $100 \mathrm{GHz}$ and $10 \mathrm{THz}$, corresponding to wavelengths from $3 \mathrm{~mm}$ in the microwave domain to $30 \mu \mathrm{m}$ in the far infrared. Specifically, THz energy corresponds to the intermolecular oscillations, which can identify and differentiate between different polymorphic crystals or crystal orientations of the same molecule. 
Terahertz radiation is currently used to determine the hydration state of living cells and biomaterials since the dielectric responses are selectively sensitive to bulk water [2]]. Any other polar liquid can also absorb $\mathrm{THz}$ radiation and can thus be used in remote sensing of any process that results from changes in the water content (WC) of the sample [1]. For instance, it is commonly applied to non-invasive inspection of the WC in leaves $[\underline{3}, \underline{4}]$, drying paint $[\underline{5}, \underline{6}]$ and to evaluate variations in WC when a healthy tissue becomes cancerous $[\underline{7}, \underline{8}]$.

The development of hydrogel biomaterials for contact lens (CL) application has been driven by the need to increase the biocompatibility of CLs as a means to extend the wearing time. Biocompatibility is dependent on multiple factors, which can be classified in 2 main categories: $i$ ) bulk parameters, such as oxygen permeability $D k$ and equilibrium WC; $i$ ) interfacial phenomena, such as wettability of the polymeric surface. In essence, biocompatibility of CLs refers to the characteristics of both bulk and surface properties when interacting with the tear film and the lens care solution.

Conventional hydrogel biomaterials for CLs were based in the synthetic biocompatible material poly(hydroxyethylmethacrylate) (PHEMA), which presents a matrix surface with hydrophilic character that allows sufficient surface wettability at the interface between the CL and the tear film. The increased equilibrium WC of the hydrogel polymeric matrix has led to the development of new PHEMA-based hydrogel materials with higher $D k$ [9]. On the other hand, the first generation of silicone hydrogel biomaterials for CLs was formulated by block polymerization of tris(trimethylsiloxy)silylpropylmethacrylate (TRIS), polydimethylsiloxane (PDMS) and dimethylacrylamide macromers [10] that resulted in higher $D k$ values than conventional hydrogel CLs. However, the hydrophobic character of the polymeric surface requires treatment to ensure wettability. This is usually achieved by increasing the WC of the hydrogel matrix without decreasing its $D k$, while also lowering the tensile modulus of the first silicone hydrogel generation materials to improve comfort $[\underline{11}, \underline{12}]$.

With regard to the evaluation of $\mathrm{WC}$ and $\mathrm{CL}$ dehydration, gravimetric [13-16] and refractometric $[\underline{17}, \underline{18}]$ methods have been widely applied to silicone and conventional hydrogel CLs for in vitro evaluation, since they can determine the dehydration rate in different environments. Wettability of silicone and conventional hydrogel materials has also been assessed by means of the contact angle of pure water and surfactant solutions for CLs preserved in different solutions $[19,20]$.

In this work, we propose a novel $\mathrm{THz}$ system for the in vitro evaluation of the dehydration process of different materials used for conventional and silicone hydrogel CLs and the influence of conditioning solutions with different surfactants and lubricant agents. The gravimetric WC and wettability of the CL polymeric surface were also assessed to validate the performance of the system. The main goal was to provide a more precise analysis of water loss considering the bulk and superficial performance of CL materials by means of a THz-based approach that, to the authors' knowledge, has not been used previously for this purpose. Furthermore, the system can contribute to a better understanding of user's wearability of hydrogel CLs as a function of the ma- 
terial, WC and the solution used to achieve the hydrated state required. The proposed technique also provides complementary and valuable information not available in the literature.

\section{Material and methods}

\subsection{Dehydration measurements}

\subsubsection{Experimental setup}

The dehydration process of CLs was evaluated with a novel THz-based system (Fig. 1), which consisted of a mechanically tuned Gunn oscillator SOM-94301313-10-SC coupled to a voltage regulator SOR-R3, and an amplitude GaAs Schottky detector SFD-753114-10SF-N from SAGE Millimeter, Inc. The Gunn oscillator was fed with $7.3 \mathrm{~V}$ and emitted a linearly polarized, free space radiation at $94 \mathrm{GHz}$ with approximately $1 \mathrm{GHz}$ frequency modulate bandwidth. Additionally, 2 SGH-26-WR10 B horn antennas from Anteral S.L. were attached to the oscillator and detector; a polarizer FPOL -MMW-20 from the same company was also placed in front of the oscillator-antenna assembly in order to maximize the signal. A NI USB-3211 acquisition card from National Instruments Corporation was incorporated to retrieve the signal detected by the sensor.

The beam propagation from the emitter to the detector was handled by a configuration of 4 Teflon $^{\mathrm{TM}}$ lenses from Thorlabs $\mathrm{GmbH}$, which included 2 collimating lenses of 100-mm focal length (L1 and L4) and two aspherical lenses with a focal length of $50 \mathrm{~mm}$ (L2 and L3). In order to guarantee the perpendicular positioning of the CL with respect to the optical path, a transparent plastic holder with a semi-A sphere of $8-\mathrm{mm}$ radius in the centre that simulated the cornea was used.

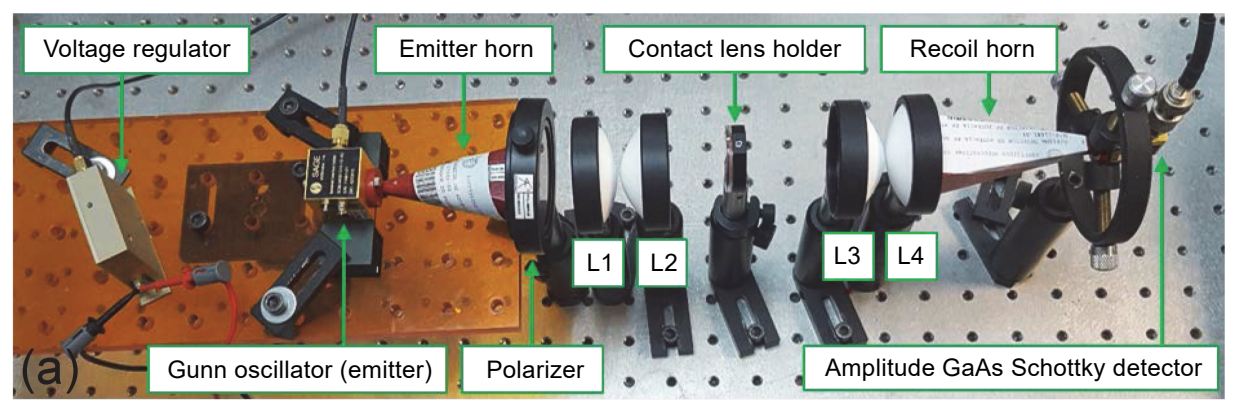

a

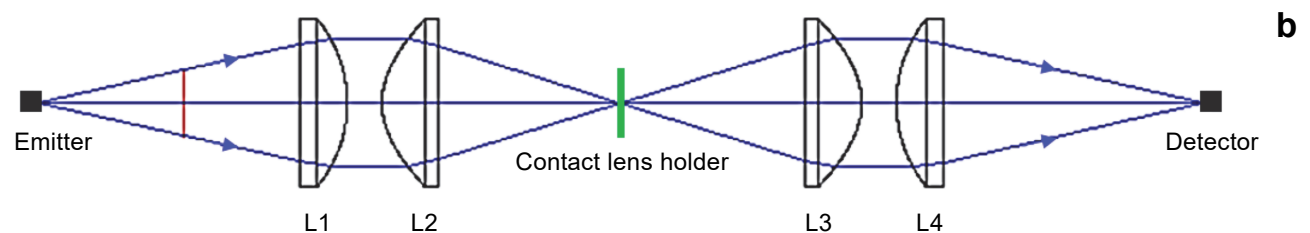

Fig. 1. General view of the setup (a) and ray tracing from the emitter to the detector (b); L1, L2, L3 and L4 refer to the four Teflon ${ }^{\mathrm{TM}}$ lenses. 


\subsubsection{Hydration index (HI)}

Dehydration was measured in terms of transmission by comparing the voltage detected with and without the CL in the optical path. This transmitted voltage was expressed as a percentage through a hydration index (HI) as follows:

$$
\mathrm{HI}=100-\frac{V_{0}-V_{i}}{V_{0}} \times 100
$$

where $V_{0}$ refers to the initial value of voltage and $V_{i}$ indicates the voltage for each individual measurement over time. The computation of each voltage value (either $V_{0}$ or $V_{i}$ ) was calculated as follows:

$$
V_{i}=1-\frac{R_{i}-\Delta R}{R_{\mathrm{H}}}
$$

where $R_{i}$ is the raw voltage value obtained when measuring the transmission of the CL and its holder, $R_{\mathrm{H}}$ is the voltage transmitted only using the holder; the influence of the holder on the total transmission is thus avoided. Moreover, dividing by $R_{\mathrm{H}}$ also benefits the minimization of the effect of environmental conditions such as variations of humidity, i.e., water vapour present in the air, which were monitored during measurements, showed fluctuations below $2 \%$. The term $\Delta R$ is calculated as $R_{n}-R_{\mathrm{H}}$ and was added to obtain HI values of zero when the signal was constant, which meant that the CL was completely dehydrated at time $n$. At this time, some CLs showed a constant signal but with values slightly above zero, which might have been caused by the inner structure of the material. In relation to the assessment of different lens care solutions, the $\Delta R$ remained constant for each CL material but was different among the solutions. In this case, the $\Delta R$ selected as a reference was the $\Delta R$ of the CL preconditioned in MilliQ ${ }^{\circledR}$ water (DW). This voltage ratio was finally subtracted from 1 to obtain a curve decreasing over time in consonance with the expected behaviour of the HI.

\subsubsection{CLs preconditioning}

With regard to the assessments of different solutions, once the CLs had been 24 hours in DW and before the $\mathrm{THz}$ measurements, they were immersed in the solution under test for 24 additional hours. Next, the residual water was eliminated with lens cleaning tissue and the sample was weighted to establish the initial mass. The time needed for these processes was about 1 minute to minimize dehydration before $\mathrm{THz}$ measurements. The dehydration was measured until the complete stabilization of the signal, indicated by constant and very low values of voltage. Each CL was tested once, but the transmitted voltage measurement was repeated at least twice, with new samples for each material and preconditioning process, in order to assure the repeatability of the HI index. Other factors also considered during the preconditioning processes were the use of silicone tweezers and nitrile gloves to avoid any contamination of the CLs. 
Room temperature and relative humidity were kept constant at $22 \pm 1^{\circ} \mathrm{C}$ and $32 \pm 2 \%$, respectively.

\subsubsection{Gravimetric determination of the WC}

The WC of different materials was gravimetrically quantified by means of a PCE-AB 100 analytical balance from PCE Instruments ${ }^{\mathrm{TM}}$, with a precision of $0.1 \mathrm{mg}$. The following formula was used to calculate the $\mathrm{WC}$ as a percentage:

$$
\mathrm{WC}=\frac{m_{0}-m_{n}}{m_{0}} \times 100 \%
$$

where $m_{0}$ refers to the initial value of mass measured just after the preconditioning process, and $m_{n}$ indicates the final mass calculated when the CL is completely dehydrated, i.e., when the $\mathrm{THz}$ signal becomes stable.

\subsection{CL materials and solutions}

Eight CLs were analysed: 5 silicone hydrogel ( $\mathrm{SiHy}$ ) and 3 conventional hydrogel (Hy), i.e., poly(hidroxyethylmethacrylate)-based, CLs (Table 1). All had the same optical power $(-4.00 \mathrm{D})$ to prevent differences among them. Concerning hydration, $3 \mathrm{CL}$ materials with high $(>50 \%)$ and 5 with low WC $(<50 \%)$ were included in the study. With regard to the material characteristics, 2 were ionic and 6 non-ionic. This sample set consisted of monthly and biweekly replacement CLs except for the etafilcon A, which is a material for daily use only.

Borate buffered saline solution was prepared using analytical grade reagents from Sigma-Aldrich and deionized ultrapure MilliQ ${ }^{\circledR}$. The BBS solution $(\mathrm{pH}=7.40 \pm 0.02$ and osmolarity of $304 \pm 0.5 \mathrm{mOsm} / \mathrm{L})$ contained boric acid $(0.01 \mathrm{M})$, sodium tetraborate decahydrate $(0.16 \mathrm{mM})$ and sodium chloride $(0.14 \mathrm{M})$. BBS solutions containing in addition hyaluronic acid sodium salt (HA, molecular weight of $1.5-1.8 \times 10^{3} \mathrm{kDa}$ ) and/or purified Poloxamer-407 (P, molecular weight $12.6 \mathrm{kDa}$ ) were also prepared. The BBS-HA, BBS-P, and BBS-HA-P solutions contained $0.01 \% \mathrm{wt} / \mathrm{vol} \mathrm{HA}, 0.2 \% \mathrm{wt} / \mathrm{vol} \mathrm{P}$ and $0.01 \% \mathrm{wt} / \mathrm{vol} \mathrm{HA}+0.2 \% \mathrm{wt} / \mathrm{vol} \mathrm{P}$, respectively. Finally, the commercial multi-purpose solution Biotrue ${ }^{\circledR}$ from Bausch + Lomb $(\mathrm{pH}=7.50 \pm 0.02$ and osmolarity equal to $285 \pm 1.0 \mathrm{mOsm} / \mathrm{L})[\underline{21}, \underline{22}]$ was also evaluated.

\subsection{Static contact angle evaluation}

The static contact angles $\theta$ of water drops on the surface of CLs were measured by means of the sessile drop method using a DSA100 drop shape analyser from KRÜSS GmbH. All measurements were also conducted at a room temperature of $22 \pm 1^{\circ} \mathrm{C}$. Prior to these measurements, the CLs were removed from the blister packages and immersed in BBS solution during two minutes, followed by the immersion in the test solution for 48 hours. 


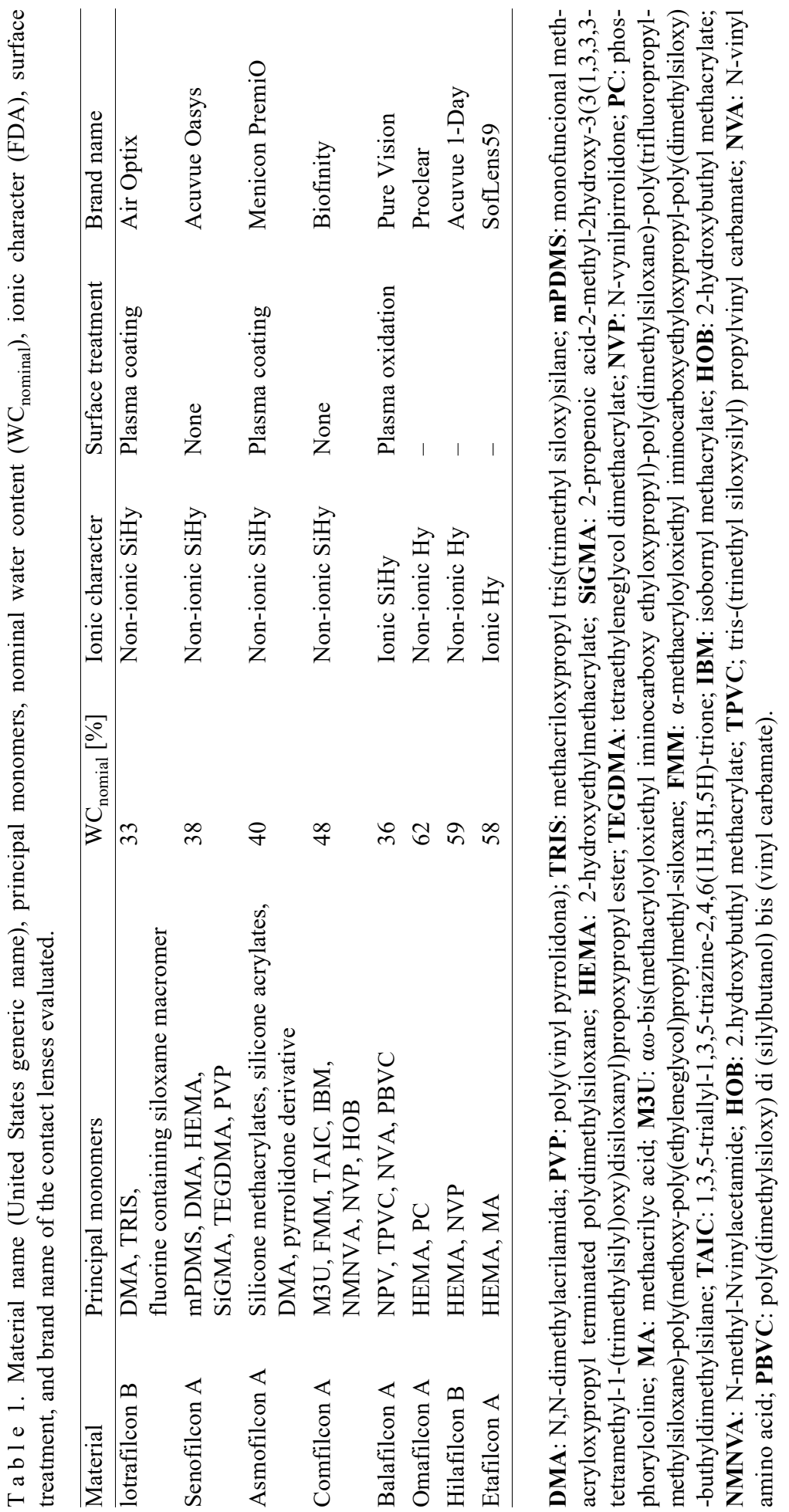


The methodology followed to obtain the contact angle measurements was based on previous works $[19$, 20]. Firstly, the CL was removed from the storage solution using silicone tweezers and repeatedly placed on a lens cleaning tissue to collect any residual surface liquid. Next, the CL was placed on a holder and a $4 \mu 1$ water drop controlled by a micrometre pass dosage was transferred to the CL surface, ensuring a total time of exposure to air of $2 \mathrm{~min}$. This process was captured in the digital video by the camera integrated in the drop shape analyser, controlled and analysed by the software DSA4. A tangent method was used to measure the contact angles. Three independent fittings were performed to provide three mean contact angles of the same drop image and the average $\left(\theta_{m}\right)$ was determined. This procedure was repeated for a second video of the same CL solution to verify the reproducibility of the measurement.

\section{Results and discussion}

\subsection{Dehydration evaluation of different $C L$ materials preconditioned in DW}

Figure 2 shows the dehydration process, indicating the HI index for the eight CLs preconditioned in DW at different times (Fig. 2a) and for $5000 \mathrm{~s}$ (Fig. 2b). The first time of $20 \mathrm{~s}$ was selected to simulate natural eye conditions, where the average tear film break up time (TFBUT) is $17 \mathrm{~s}$, approximately. On the other hand, between 4000 and $5000 \mathrm{~s}$, the voltage of all CLs remained constant and at minimum values; therefore, $5000 \mathrm{~s}$ were thus assumed to be enough for all CLs to dehydrate completely.

The dehydration process within the first time interval $(0 \mathrm{~s}, 20 \mathrm{~s})$ in Fig. $2 \mathbf{b}$ revealed that the HI was not dependent on the tested WC of the CL material (Table 2). Six CL materials showed a mean dehydration under $2 \%$ (balafilcon $\mathrm{A}$, comfilcon $\mathrm{A}$, omafilcon $\mathrm{A}$, hilafilcon $\mathrm{B}$ and lotrafilcon $\mathrm{B}) ; 2$ other materials exhibited values between $2 \%$ and $3 \%$
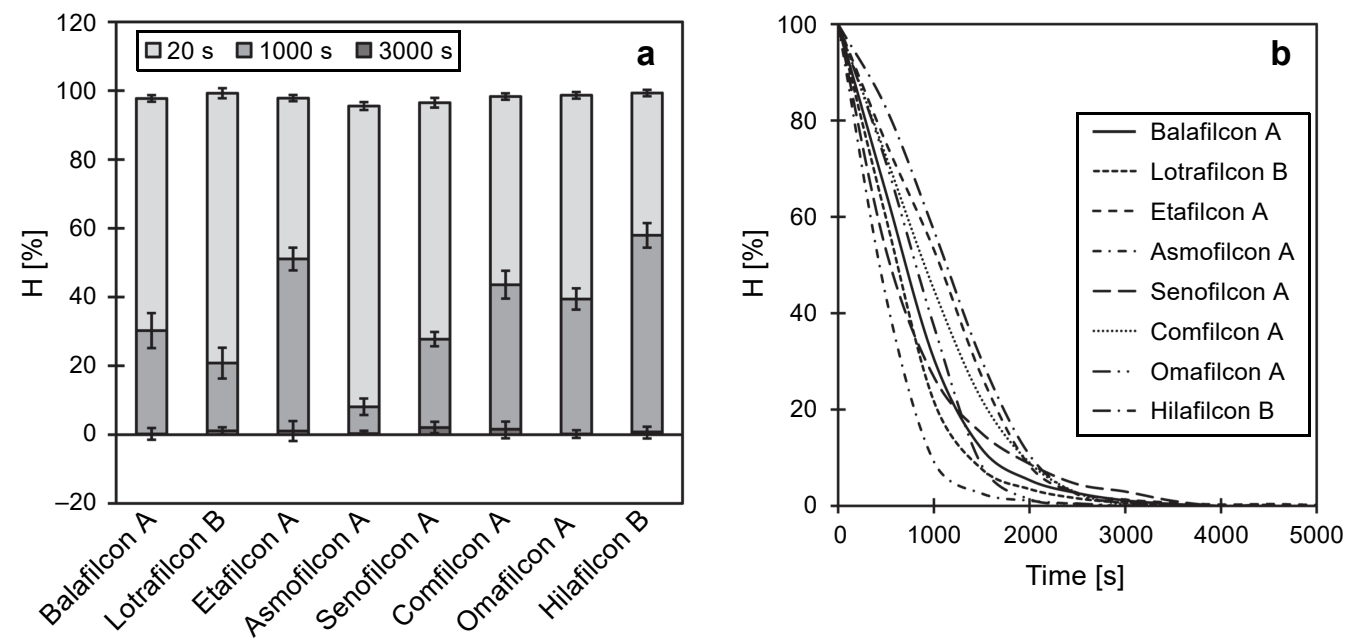

Fig. 2. Dehydration evolution for the eight contact lenses conditioned in MilliQ ${ }^{\circledR}$ water (DW) analysed at different times (a) and during $5000 \mathrm{~s}(\mathbf{b})$. 
$\mathrm{T}$ a b $1 \mathrm{e}$ 2. Nominal and tested $\mathrm{WC}$ of the eight contact lenses. Both are expressed as a percentage.

\begin{tabular}{lll}
\hline Material & $\mathrm{WC}_{\text {nominal }}$ & $\mathrm{WC}_{\text {tested }} \pm \mathrm{SD}$ \\
\hline Lotrafilcon B & 33 & $32.7 \pm 0.6$ \\
Senofilcon A & 38 & $37.0 \pm 0.0$ \\
Asmafilcon A & 40 & $41.3 \pm 1.2$ \\
Comfilcon A & 48 & $45.0 \pm 1.7$ \\
Balafilcon A & 36 & $36.3 \pm 0.6$ \\
Omafilcon A & 62 & $61.7 \pm 1.2$ \\
Hilafilcon B & 59 & $57.7 \pm 4.6$ \\
Etafilcon A & 58 & $58.0 \pm 1.7$ \\
\hline
\end{tabular}

(etafilcon A and senofilcon A); and only one led to a mean dehydration of almost 5\% (asmofilcon A). In the second time interval $(20 \mathrm{~s}, 1000 \mathrm{~s})$, the dehydration was more dependent on the tested WC and the CL polymeric matrix material. All Hy CLs had dehydration rates lower than SiHy materials, as expected due to their higher WC, with the exception of comfilcon A. The three Hy CLs and comfilcon A (SiHy) showed a $\mathrm{HI} \geq 40 \%$ at $t=1000 \mathrm{~s}$. Among all Hy CLs evaluated, the hilafilcon A material presented the lowest dehydration rate, i.e., the highest $\mathrm{HI}$ in this range; the ionic character did not seem to influence the dehydration process. In the case of SiHy materials, comfilcon A reached the highest HI values up to $2000 \mathrm{~s}$, which corresponds to its inherent wettability as a third generation SiHy CL [10]. The remaining SiHy materials showed $\mathrm{HI}<40 \%$ at $t=1000 \mathrm{~s}$. The ionic material balafilcon A exhibited the slowest dehydration rate, even though its dehydration behaviour is very similar to that of lotrafilcon B, both belonging to the first generation of SiHy CLs. Although the HI decrease of senofilcon A was initially faster, it presented the slowest dehydration decay beyond $1000 \mathrm{~s}$ ( 3 rd time interval) due to the PVP internal wetting agent. Beyond $3000 \mathrm{~s}$, most CL materials were completely dehydrated. The materials with the longest dehydration times were etafilcon B and senofilcon A, while asmofilcon A and omafilcon A presented the shortest dehydration times, with $\mathrm{HI} \sim 0$ at $2500 \mathrm{~s}$ and $2750 \mathrm{~s}$, respectively.

Table 2 shows the $\mathrm{WC}$ provided by the manufacturers $\left(\mathrm{WC}_{\text {nominal }}\right)$ and the mean and standard deviation (SD) values of WC experimentally measured as previously described in Eq. (3) $\left(\mathrm{WC}_{\text {tested }}\right)$ for the eight CLs and calculated at $t=5000 \mathrm{~s}$. A good Pearson correlation was obtained between both $\mathrm{WC}_{\text {tested }}$ and $\mathrm{WC}_{\text {nominal }}$ values $\left(R^{2}=0.9854\right.$, $p<0.0001$ ), with a decreasing tendency in the $\mathrm{WC}_{\text {tested }}$ values for the CLs conditioned in DW respect to the $\mathrm{WC}_{\text {nominal }}$ values measured in standard saline solution (following ISO 18369-3:2017 for measuring methods in ophthalmic optics).

In general, the THz evaluation showed good inter-blink hydration since the mean $\mathrm{HI}$ of all CL materials was above $95 \%$ during the first $20 \mathrm{~s}$. Because of their slower dehydration decay, hilafilcon B, etafilcon A and comfilcon A were shown to be the most comfortable materials for long-time use. These results agreed with other studies where conventional hydrogel materials also presented lower dehydration decay than silicone -containing hydrogels [15] and CLs of higher WC dehydrated at a slower rate [23]. 


\subsection{Dehydration evaluation of CLs preconditioned in different lens care solutions}

Additionally, the dehydration of balafilcon A and senofilcon A materials was studied after preconditioning the CLs in the different prepared solutions and in the commercial Biotrue ${ }^{\circledR}$ solution. Balafilcon A and senofilcon A CLs were chosen to account for materials with similar WC belonging to different $\mathrm{SiHy}$ generations and with different ionic character. The objective of this second test was to study the behaviour of CL dehydration when using solutions with different lubricant or wetting agents.

The latest formulations of multipurpose CL solutions [24] try to mimic the physicochemical properties of natural tear film $(\mathrm{pH}$, osmolarity, viscosity and surface tension) [ $\underline{25}-27]$. This is the case of the Biotrue ${ }^{\circledR}$ and some of the prepared solutions, which incorporate HA and poloxamers or poloxamines agents. HA is a natural polysaccharide present in biological fluids and tissues, including human tears, that is commonly used in CL solutions for its wetting and lubricant properties. Poloxamers and poloxamines are non-ionic surfactants [28].

Figures 3 and 4 show the evolution of the HI index of both materials conditioned in different solutions at different times and during $5000 \mathrm{~s}$, respectively. In both figures the dehydration in MilliQ ${ }^{\circledR} \mathrm{DW}$ was also included as reference.

The assessments of balafilcon A revealed that for the first time interval $(0 \mathrm{~s}, 20 \mathrm{~s})$, the mean HI was above $96 \%$ for all solutions. The highest mean HI was obtained in the case of DW, BBS and the multipurpose Biotrue ${ }^{\circledR}$ solutions (Fig. 3a). In the second interval (20 s, $1000 \mathrm{~s})$, the DW led to higher mean HI than BBS and both of them presented higher mean $\mathrm{HI}$ than the rest of solutions (Figs. 3a and 4a). Moreover, the decrease of the HI was constant for the Biotrue ${ }^{\circledR}$, BBS-HA-P, BBS-HA and BBS-P solutions until around $95 \%$ of water had been lost, presenting a change in the dehydration profile respect to that of DW. For all solutions and DW, balafilcon A became completely de-
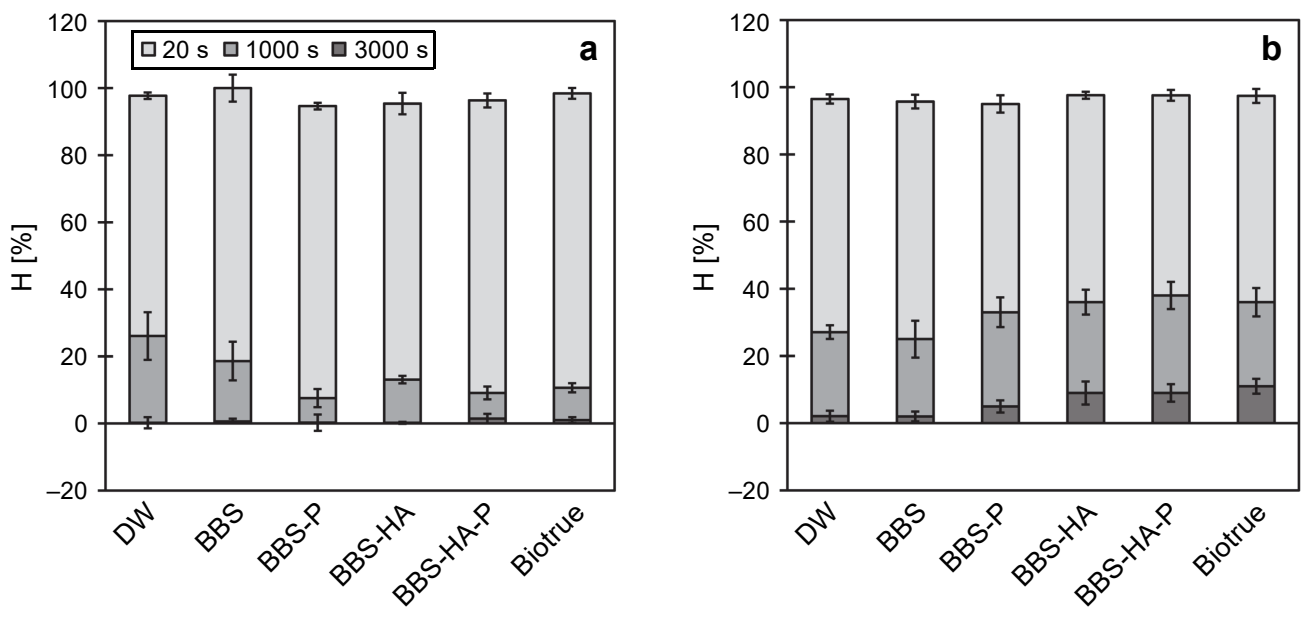

Fig. 3. Dehydration evolution of the balafilcon A (a) and the senofilcon A (b) contact lens materials conditioned in different lens care solutions at different times. 

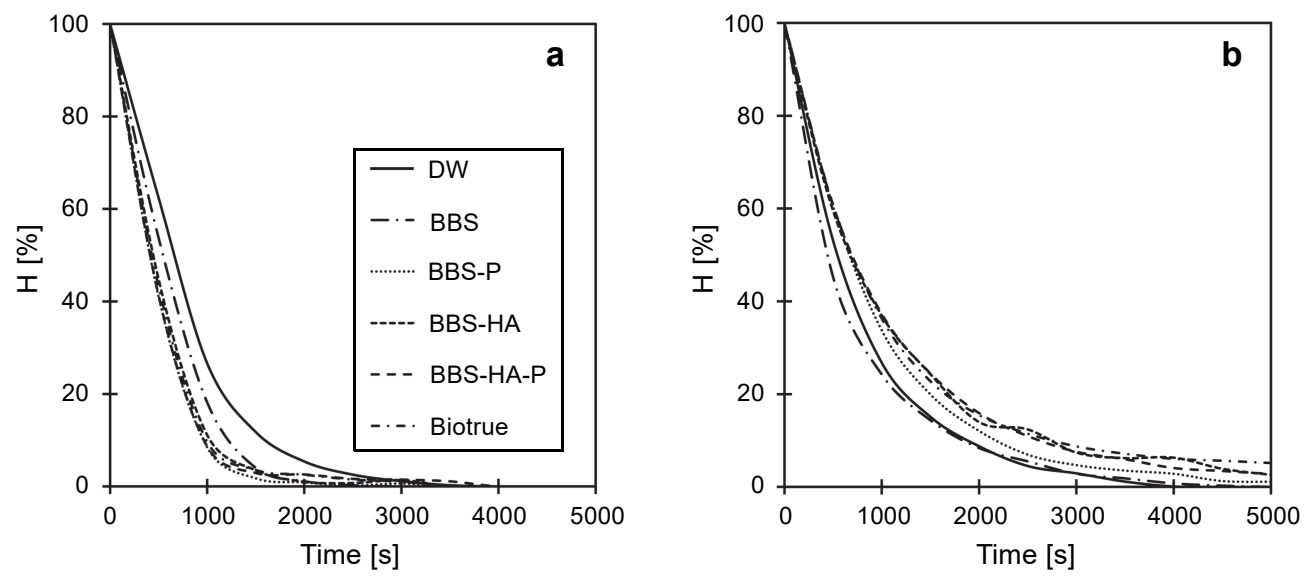

Fig. 4. Dehydration evolution of the balafilcon A (a) and the senofilcon A (b) contact lens materials conditioned in different lens care solutions during $5000 \mathrm{~s}$.

$\mathrm{T}$ a b $1 \mathrm{e}$ 3. Tested $\mathrm{WC}$ in percentage and static contact angles $\left(\theta_{m}\right.$, degrees) of the balafilcon $\mathrm{A}$ and the senofilcon A contact lenses for the different lens care solutions.

\begin{tabular}{lllll}
\hline \multirow{2}{*}{ Solution } & Balafilcon A & \multicolumn{3}{l}{ Senofilcon A } \\
\cline { 2 - 5 } $\mathrm{WC}_{\text {tested }} \pm \mathrm{SD}$ & $\theta_{m} \pm \mathrm{SD}$ & $\mathrm{WC}_{\text {tested }} \pm \mathrm{SD}$ & $\theta_{m} \pm \mathrm{SD}$ \\
\hline DW & $36.3 \pm 0.6$ & $29.9 \pm 2.6$ & $37.0 \pm 0.0$ & $97.2 \pm 1.2$ \\
BBS & $35.0 \pm 0.0$ & $33.6 \pm 1.3$ & $38.0 \pm 0.0$ & $55.1 \pm 1.7$ \\
BBS-P & $33.5 \pm 2.4$ & $31.0 \pm 0.8$ & $36.5 \pm 0.7$ & $36.8 \pm 2.1$ \\
BBS-HA & $36.5 \pm 0.7$ & $36.7 \pm 1,9$ & $38.0 \pm 0.0$ & $26.8 \pm 1.1$ \\
BBS-HA-P & $35.0 \pm 0.0$ & $25.1 \pm 0.6$ & $36.0 \pm 0.0$ & $39.0 \pm 2.6$ \\
Biotrue $^{\circledR}$ & $39.0 \pm 0.0$ & $26.6 \pm 0.3$ & $38.0 \pm 0.0$ & $39.5 \pm 1.6$ \\
\hline
\end{tabular}

hydrated around $3000 \mathrm{~s}$ (Fig. 4a) and the values of WC tested were almost identical to the nominal WC (Table 3), except for the Biotrue ${ }^{\circledR}$ that presented a value slightly higher and for the BBS-P slightly lower. This allowed us to conclude that the total equilibrium WC of balafilcon A is not much affected by the presence of surfactant or lubricant agents in the lens care solutions. However, in contrast with DW, the osmolarity change and the addition of these agents in the preconditioning solution affected the kinetics of the dehydration process increasing the dehydration rate. This phenomenon was less noticeable during the first $20 \mathrm{~s}$ of the dehydration process since a similar performance with a decrease of the HI below $6 \%$ was observed for all solutions.

On the other hand, the tests for senofilcon A in the interval $(0 \mathrm{~s}, 20 \mathrm{~s})$ showed that the mean HI was above $94 \%$ for all solutions. The highest mean HI values were obtained for the BBS-HA, BBS-HA-P and Biotrue ${ }^{\circledR}$ (Fig. 3b). In the interval (20 s, $\left.1000 \mathrm{~s}\right)$ BBS-P, BBS-HA, BBS-HA-P, and Biotrue ${ }^{\circledR}$ solutions produced very similar dehydration curves (Fig. 4b), always above those of DW and BBS, with higher mean HI values 
at $t=1000 \mathrm{~s}$ (Fig. 3b). Senofilcon A became completely dehydrated around $4000 \mathrm{~s}$ in the case of DW and BBS, and at $4500 \mathrm{~s}$ in the case of BBS-P; in all cases, the dehydration process lasted longer than that of balafilcon A. For the remaining solutions, an apparent residual $\mathrm{HI}$ value under $5 \%$ was observed when the signal was considered stable (5000 s). However, the values of WC tested at $5000 \mathrm{~s}$ (Table 3 ) indicated that in all cases the senofilcon A CLs were completely dehydrated since these values essentially coincided with the nominal WC. On the one hand, it can be concluded that the total equilibrium WC of senofilcon A behaves similar to that found for balafilcon A, i.e., is almost independent from the presence of these agents. On the other hand, the THz data transmitted by the senofilcon A material in a dehydrated state seemed to be affected by the presence of HA, $\mathrm{P}$ and/or poloxamine in the BBS solution, since a remaining amount of $\mathrm{HI}$ persisted up to $5000 \mathrm{~s}$. It probably occurred because these agents were adsorbed on the polymeric matrix surface during the preconditioning process. In order to obtain more in depth information about this fact, the contact angle of both balafilcon A and senofilcon A conditioned in DW and in the different care solutions was measured.

The gravimetric determination of the WC and the static contact angle were also experimentally evaluated for this second set of measurements. The contact angles shown in Table 3 indicate the effect of the lens care solutions on the polymeric surface wettability of the CLs. As previously mentioned, the balafilcon A material incorporates a surface treatment to increase its wettability, which was confirmed by the contact angle values when conditioned in DW or BBS. The presence of only one agent (HA or P) in the BBS solution did not produce noticeable changes in surface wettability. However, the inclusion of two agents as in the BBS-HA-P (HA and P) and the Biotrue ${ }^{\circledR}$ solutions (HA and poloxamine) achieved an improvement of surface wettability. Comparing the contact angle measurements with the mean $\mathrm{HI}$ values in the interval time $(0 \mathrm{~s}, 20 \mathrm{~s})$ in Figs. 3a for the 4 solutions containing surfactant and lubricant agents, the BBS-HA and Biotrue ${ }^{\circledR}$ showed slightly higher HI values. The selection of the most suitable solution for balafilcon A is complex because this material showed a very good hydration performance when using only DW and BBS. However, the wettability of the surface is also important in the ocular environment, since it determines how the tear film is spread over the surface of the CL. Considering the results for these two parameters, BBS-HA-P and Biotrue ${ }^{\circledR}$ would be the best solutions due to their high mean HI value during the first $20 \mathrm{~s}$ and the low contact angles, although long-term HI values were below those of DW and BBS.

Senofilcon A incorporates PVP to increase the hydrophilicity of the surface [2]. Despite this fact, the contact angle of senofilcon A conditioned in DW and BBS solution indicated a low hydrophilic surface character (Table 3). Consequently, the presence of wetting or lubricant agents in the BBS solution improved the wettability considerably, especially in the case of HA. On the other hand, the hydrophobic character of the senofilcon A surface was shown to depend on the non-ionic surfactant agents added to the lens care solutions such as BBS-P, BBS-HA-P and Biotrue ${ }^{\circledR}$. This behaviour indicated that non-ionic surfactants $\mathrm{P}$ and poloxamine were less effective than using HA without surfactants. The contact angles for senofilcon A also cor- 
related with the mean $\mathrm{HI}$ values during the first $20 \mathrm{~s}$; the lower the static contact angle, the higher the HI values. In addition, the stronger adsorption of HA and HA $+\mathrm{P}$ or $\mathrm{HA}+$ poloxamine on the senofilcon A surface correlated with the residual water content that was observed for these solutions at $5000 \mathrm{~s} \mathrm{(Fig.} \mathrm{4b).} \mathrm{Considering} \mathrm{the} \mathrm{results}$ of HI and contact angle, the most suitable solution for senofilcon A CLs is the BBS-HA, since it showed the best hydration throughout time and wettability, with a contact angle below $30^{\circ}$.

\section{Conclusions}

A THz system was developed to evaluate the dehydration of conventional and silicone hydrogel CLs and to analyse the performance of different lens care solutions by means of a THz-based system. This presents a significant extension of existing works in this field as THz technology is used for the first time for the CLs study. Based on THz measurements, a new expression for the quantification of the hydration state of CLs was defined. The results of the new system correlated with results obtained with traditional methods.

The assessment of the in vitro dehydration process of different CL materials conditioned in MilliQ ${ }^{\circledR}$ DW revealed different dehydration kinetics in the first stages $(0 \mathrm{~s}, 20 \mathrm{~s})$ compared to the dehydration kinetics at longer times $(20 \mathrm{~s}, 5000 \mathrm{~s})$. Although the dehydration profile of the different materials was similar within the Hy group and among SiHy CLs of the same generation, each dehydration profile presented specific features that could be used as a fingerprint for material identification.

The study of the effect of different solutions on the in vitro dehydration profile of CLs was analysed for the balafilcon A and senofilcon A materials. The solutions used were based on BBS in combination with wetting and/or lubricant components. For each solution, a correlation was observed between the measurements of dehydration by means of the HI and of the static contact angle.

To conclude, the THz-based system contributed to improve the accuracy in the study of hydrogel CL materials and their interaction with lens care solutions. The system evaluated the performance of biomaterials and solutions in terms of dehydration and can be a useful technique to evaluate new hydrogel biomaterials and their behaviour prior to in vivo tests.

Acknowledgements - This work was supported by the Spanish Ministry of Economics and Competitiveness (MINECO) under the grant DPI2014-56850-R and the European Union.

\section{References}

[1] Xiang Yang, Xiang Zhao, Ke Yang, Yueping Liu, Yu Liu, Weiling Fu, Yang Luo, Biomedical applications of terahertz spectroscopy and imaging, Trends in Biotechnology 34(10), 2016, pp. 810-824, DOI: $10.1016 / \mathrm{j}$. tibtech.2016.04.008.

[2] Shiraga K., Suzuki T., Kondo N., Tanaka K., Ogawa Y., Hydration state inside HeLa cell monolayer investigated with terahertz spectroscopy, Applied Physics Letters 106(25), 2015, article ID 253701, DOI: $10.1063 / 1.4922918$. 
[3] Gente R., Косн M., Monitoring leaf water content with THz and sub-THz waves, Plant Methods 11, 2015, article ID 15, DOI: 10.1186/s13007-015-0057-7.

[4] Breitenstein B., Scheller M., Shakfa M.K., Kinder T., Müller-Wirts T., Koch M., Selmar D., Introducing terahertz technology into plant biology: a novel method to monitor changes in leaf water status, Journal of Applied Botany and Food Quality 84(2), 2011, pp. 158-161.

[5] YAO-CHUN SHEN, Terahertz sensor for noncontact and non-destructive inspection of automotive paints, International Journal of Sensor Networks and Data Communications 4(1), 2014, article ID e103, DOI: $10.4303 / 2090-4886.1000 \mathrm{e} 103$.

[6] Fukunaga K., Hosako I., Innovative non-invasive analysis techniques for cultural heritage using terahertz technology, Comptes Rendus Physique 11(7-8), 2010, pp. 519-526, DOI: 10.1016/j.crhy. 2010.05.004.

[7] Yu C., Fan S., Sun Y., Pickwell-MacPherson E., The potential of terahertz imaging for cancer diagnosis: A review of investigations to date, Quantitative Imaging in Medicine and Surgery 2(1), 2012, pp. 33-45.

[8] Rahman A., Rahman A.K., Rao B., Early detection of skin cancer via terahertz spectral profiling and 3D imaging, Biosensors and Bioelectronics 82, 2016, pp. 64-70, DOI: 10.1016/j.bios.2016. $\underline{03.051 .}$.

[9] Nicolson P.C., Vogt J., Soft contact lens polymers: an evolution, Biomaterials 22(24), 2001, pp. 3273-3283, DOI: 10.1016/S0142-9612(01)00165-X.

[10] ЈАСов J.T., Biocompatibility in the development of silicone-hydrogel lenses, Eye Contact Lens 39(1), 2013, pp. 13-19, DOI: 10.1097/ICL.0b013e31827dbb00.

[11] Tighe B.T., A decade of silicone hydrogel development: surface properties, mechanical properties, and ocular compatibility, Eye and Contact Lens 39(1), 2013, pp. 4-12, DOI: 10.1097/ICL.0b013e31 $\underline{8275452 b .}$.

[12] Hutter J.C., Green J.A., Eydelman M.B., Proposed silicone hydrogel contact lens grouping system for lens care product compatibility testing, Eye and Contact Lens 38(6), 2012, pp. 358-362, DOI: 10.1097/ICL.0b013e318260c959.

[13] Jones L., May C., NAZAR L., Simpson T., In vitro evaluation of the dehydration characteristics of silicone hydrogel and conventional hydrogel contact lens materials, Contact Lens and Anterior Eye 25(3), 2002, pp. 147-156, DOI: 10.1016/S1367-0484(02)00033-4.

[14] González-MéiJome J.M., LóPez-Alemany A., Almedia J.B., Parafita M.A., Dynamic in vitro dehydration patterns of unworn and worn silicone hydrogel contact lenses, Journal of Biomedical Materials Research Part B: Applied Biomaterials 90B(1), 2009, pp. 250-258, DOI: 10.1002/jbm. b.31279.

[15] Krysztofiak K., Szyczewski A., Study of dehydration and water states in new and worn soft contact lens materials, Optica Applicata 44(2), 2014, pp. 237-250, DOI: 10.5277/oa140206.

[16] Rajchel D., Krysztofiak K., Szyczewski A., Influence of sodium hyaluronate on dehydration and water distribution in soft contact lenses, Optica Applicata, 46(3), 2016, pp. 483-496, DOI: $10.5277 /$ oa160314.

[17] Morgan P.B., Efron N., Morgan S.L., Little S.A., Hydrogel contact lens dehydration in controlled environmental conditions, Eye and Contact Lens 30(2), 2004, pp. 99-102, DOI: 10.1097/01.ICL.000 00118532.90284 .09 .

[18] Alemany A.L., Refojo M.F., Comparative study of the hydration of hydrophilic contact lenses by refractive index and gravimetry, CLAO J 26(4), 2000, pp. 200-203.

[19] Ketelson H.A., Meadows D.L., Stone R.P., Dynamic wettability properties of a soft contact lens hydrogel, Colloids and Surfaces B: Biointerfaces 40(1), 2005, pp. 1-9, DOI: 10.1016/j.colsurfb.2004. $\underline{07.010 .}$.

[20] Maldonado-Codina C., Morgan P.B., In vitro water wettability of silicone hydrogel contact lenses determined using the sessile drop and captive bubble techniques, Journal of Biomedical Materials Research Part A 83A(2), 2007, pp. 496-502, DOI: 10.1002/jbm.a.31260.

[21] Supplement: Bringing new inspiration to contact lens care, Contact Lens Spectrum, September 2010. 
[22] Liu X.M., Harmon P.S., Maziarz E.P., Rah M.J., Merchea M.M., Comparative studies of hyaluronan in marketed ophthalmic products, Optometry and Vision Science 91(1), 2014, pp. 32-38, DOI: 10.1097/OPX.0000000000000100.

[23] Efron N., Young G., Dehydration of hydrogel contact lenses in vitro and in vivo, Ophthalmic and Physiological Optics 8(3), 1988, pp. 253-256, DOI: 10.1111/j.1475-1313.1988.tb01055.x.

[24] Dalton K., Subbaraman L.N., Rogers R., Jones L., Physical properties of soft contact lens solutions, Optometry and Vision Science 85(2), 2008, pp. 122-128, DOI: 10.1097/OPX.0b013e318162261e.

[25] Benelli U., Nardi M., Posarelli C., Albert T.G., Tear osmolarity measurement using the TearLab ${ }^{\mathrm{TM}}$ Osmolarity System in the assessment of dry eye treatment effectiveness, Contact Lens and Anterior Eye 33(2), 2010, pp. 61-67, DOI: 10.1016/j.clae.2010.01.003.

[26] Nagyová B., Tiffany J.M., Components responsible for the surface tension of human tears, Current Eye Research 19(1), 1999, pp. 4-11, DOI: 10.1076/ceyr.19.1.4.5341.

[27] Gouveia S.M., Tiffany J.M., Human tear viscosity: an interactive role for proteins and lipids, Biochimica et Biophysica Acta 1753(2), 2005, pp. 155-163, DOI: 10.1016/j.bbapap.2005.08.023.

[28] Moghimi S.M., Hunter A.C., Poloxamers and poloxamines in nanoparticle engineering and experimental medicine, Trends in Biotechnology 18(10), 2000, pp. 412-420, DOI: 10.1016/S0167-7799(00) $\underline{01485-2 .}$ 